\title{
Serpin A1 derivatives as collagen turnover modulators for cosmeceutical uses
}

\author{
Fosca Errante ${ }^{1}$, Simona Pascarella ${ }^{2}$, Caterina Cipriani ${ }^{2}$, Lisa Giovannelli ${ }^{2}$, Anna Maria Papini $^{2}$, Paolo Rovero $^{2}$ \\ ${ }^{1}$ University of Florence and Espikem srl, Prato, Italy \\ ${ }^{2}$ University of Florence, Italy
}

https://doi.org/10.17952/35EPS.2018.226

\section{Introduction}

Collagen type I is the archetype above all collagens and it is the main component of connective tissue. Alterations of the delicate balance of its synthesis and degradation lead to various pathophysiological conditions, such as osteogenesis imperfecta, Ehlers-Danlos Syndrome, inflammatory conditions and skin aging. An increased degradation of collagen type I has been shown in chronic wounds and photo-aged skin. Serine proteases, together with matrix metalloproteinases (MMPs), are the main enzymes responsible for collagen degradation within the extracellular matrix (ECM). The physiological inhibitors of these two protein families are serpins and TIMPs, respectively. Serpin A1 is one of the components of the serpins family and is involved in modulating wound healing, cell proliferation, and procollagen production. Among the reported activities, many, such as the wound healing ability, reside in the C-terminal portion of the protein, i.e. region 393-418, characterized by a $\beta$-turn structure.

Starting from these evidences, we previously performed a structure-activity relationship study on Serpin A1 C-terminal portion and identified a 10-mer sequence, named SA1-III, which is active in increasing collagen concentration in cultured human dermal fibroblasts. We subsequently decided to investigate whether a shorter sequence of the SA1-III peptide could maintain this promising activity, in order to understand its role in collagen turnover modulation. For this reason, we synthetized four overlapping peptides derived from SA1-III sequence and measured collagen type I concentration in treated fibroblasts by Western Blot analysis, keeping into account both soluble collagen secreted by fibroblasts in the culture medium and the newly-synthesized collagen forms present inside the cells.

\section{Results and Discussion}

We prepared SA1-III and its overlapping peptides by solid-phase peptide synthesis (SPPS) with the Fmoc/tBu approach on a $0.48 \mathrm{mmol} / \mathrm{g}$ Rink-Amide AM resin. All the sequences were synthetized with C-terminal amidation and $\mathrm{N}$-terminal acetylation to mimic a peptide bond. Crude peptides were purified by semipreparative HPLC, using a reverse-phase C18 column. After lyophilization, all the sequences were characterized by analytical HPLC and electro-spray ionization mass spectrometry. All peptides were used for in vitro studies on cell cultures derived from neonatal human dermal fibroblasts (NHDFs) at passages from 6 to 9 , in order to determine the concentrations of collagen type I produced in the presence of each peptide in comparison with the basal concentration of collagen, measured in untreated fibroblasts. We used TGF- $\beta 1$ as a positive control for its well-known ECM remodeling properties.

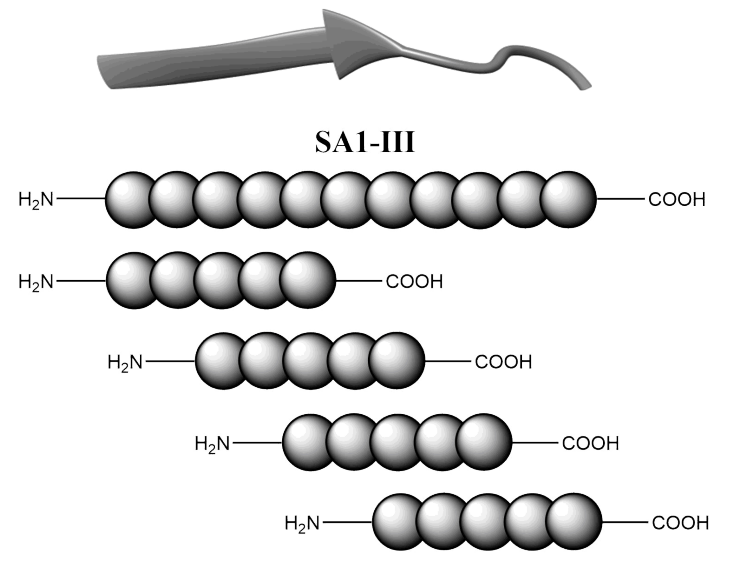

Figure 1: Overlapping analogues derived from SA1-III sequence. 
The evaluation of collagen type I concentrations was obtained by Western Blot analysis on culture medium conditioned by fibroblasts treated with the peptides in study for 48 hours, and on cell lysates as well. This immunoenzymatic technique allows to distinguish between different collagen type I forms in the membranes obtained after gel blotting, in particular those related to procollagen and to collagen degraded by the proteases released by fibroblasts in the culture medium. Our experiments show that all the shorter sequences tested display an activity comparable to that of their precursor SA1-III. In fact, all peptides induce an increase in procollagen concentration (170-150 kDa) both in cell lysates and in the media, and, most importantly, we observe in all cases a decrease of the bands related to collagen degradation fragments (100-75-60 kDa). We observed a significant (ANOVA, $\mathrm{p}<0.05$ ) activity of SA1-III and of one of its analogues in cell lysates. These data suggest that the mechanism of action of SA1-III and of the derived peptides is the modulation of collagen degradation mediated by endogenous enzymes. To confirm this result, we also tested MMP-2 and MMP-9 activity in presence and in absence of SA1-III with zymography studies; these preliminary data show enzyme activity reduction.
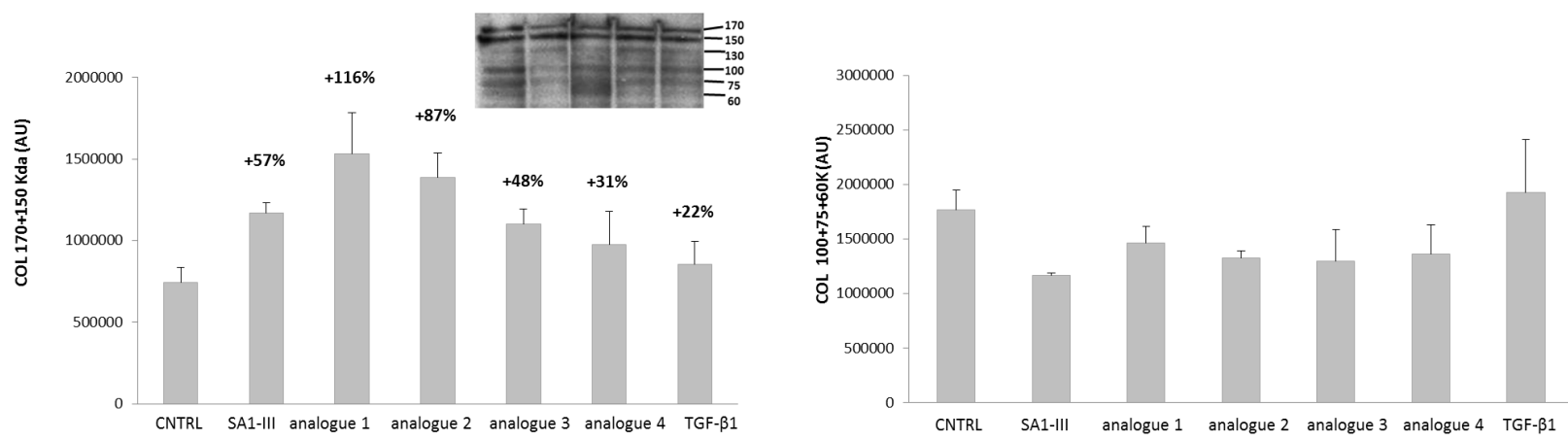

Figure 2: Increased procollagen concentration (on the left) and decreased degradation fragments (on the right) observed for SA1-III and its analogues in fibroblasts culture medium.

SA1-III was then used to formulate a cosmeceutical face cream that might be useful in all those conditions in which collagen degradation is increased, i.e. as an anti-aging factor. The claimed efficacy of this cream was demonstrated with an ultrasonographic evaluation of the cheek skin density in a small cohort of volunteers, before and after a 21-day treatment with the cream. The results clearly indicate a statistically significant increase of skin density after the treatment, thus confirming the efficacy of the cream.

\section{References}

[1] Caley M.P. et al., "Metalloproteinases and Wound Healing," Adv Wound Care (New Rochelle), vol. 4, pp. 225-234, 2015.

[2] Pillai S. et al., "Ultraviolet radiation and skin aging: roles of reactive oxygen species, inflammation and protease activation, and strategies for prevention of inflammation-inducedmatrix degradation - a review," Int J 34 of Cosmet Sci, vol. 27, pp. 17-34, 2005.

[3] Dabbagh K. et al., "Alpha-1-antitrypsin stimulates fibroblast proliferation and procollagen productionand activates classical MAP kinase signalling pathways,” J Cell Physio, vol. 186, no. 1, pp. 73-81, 2001.

[4] Congote L.F. et al., "Comparison of the effects of serpin A1, a recombinant serpin A1-IGF chimera and serpin A1 C-terminal peptide on wound healing," Peptides, vol. 29, no. 1, pp. 39-46, 2008.

[5] Pascarella S. et al., "Serpin A1 C-Terminal Peptides as Collagen Turnover Modulators," ChemMedChem, vol. 11, no. 16, pp. 1850-1855, 2016.

[6] Cipriani C. et al., "Serpin A1 and the modulation of type I collagen turnover: Effect of the C-terminal peptide 409-418 (SA1-III) in human dermal fibroblasts,” Cell Biol Int, vol. 42, no. 10, pp. 1340-1348, 2018. 